\title{
A EXPERIÊNCIA DA TARIFA SOCIAL DE ÁGUA E ESGOTO EM MINAS GERAIS
}

\section{THE EXPERIENCE OF THE SOCIAL TARIFF OF WATER AND SANITATION IN MINAS GERAIS}

\author{
Calebe Rodrigues Soares Santos ${ }^{(1)}$ \\ Engenheiro Ambiental pela Universidade Federal de Minas Gerais (UFMG). Mestrando do Programa de Pós Graduação \\ em Saneamento, Meio Ambiente e Recursos Hídricos da UFMG. \\ Paula Rafaela Silva Fonseca ${ }^{(2)}$ \\ Engenheira Ambiental e Sanitarista pela Universidade de Juiz de Fora (UFJF). Mestra, e atualmente Doutoranda pelo \\ Programa de Pós Graduação em Saneamento, Meio Ambiente e Recursos Hídricos da UFMG. \\ Natália de Cássia Onuzik ${ }^{(3)}$ \\ Nutricionista pela Universidade Federal de Ouro Preto (UFOP), Mestra em Saúde e Nutrição pela UFOP. Doutoranda \\ pelo Programa de Pós Graduação em Saúde Coletiva do Instituto René Rachou, Fiocruz Minas. \\ Pedro Américo B. de Oliveira ${ }^{(4)}$ \\ Advogado, graduado em Direito e pós-graduado em Direito Público pelo Centro Universitário Newton Paiva. Procurador \\ municipal de Igarapé-MG. \\ Rômulo José Soares Miranda ${ }^{(5)}$ \\ Economista pela Universidade Federal de Viçosa (UFV). Graduado em Ciências Contábeis pela Pontifícia Universidade \\ Católica de Minas Gerais (PUC-MG). Mestre em Economia pela UFV. Analista fiscal e de regulação da ARSAE-MG
}

E-mail ${ }^{(1)}$ : calebe12@ufmg.br.

\section{RESUMO}

Este estudo de abordagem quantitativa teve como objetivo avaliar o Programa de Tarifa Social (PTS) para Água e Esgoto nos municípios atendidos pela COPASA em Minas Gerais em termos de eficácia e eficiência. Em termos de eficiência, observou-se uma progressão no IITS entre 2015 e 2018, e uma queda entre 2019 e 2020. Em relação à eficácia, entendese que as metas e objetivos propostos caminham para a implementação progressiva da tarifa social nos municípios mineiros atendidos pela COPASA.

\begin{abstract}
This quantitative approach study had as objective to evaluate the Social Tariff Program (PTS) for water and sanitation in the municipalities served by COPASA in Minas Gerais in terms of effectiveness and efficiency. In terms of efficiency, there was a progression in IITS between 2015 and 2018, and a drop between 2019 and 2020. Regarding effectiveness, it is understood that the proposed goals and objectives are moving towards the progressive implementation of the social tariff in Minas Gerais municipalities attended by COPASA.
\end{abstract}

Palavras-chave: Universalização do Saneamento. Índice de implementação da tarifa social. Abastecimento de água e esgotamento sanitário.

Key words: Universalization of sanitation. Social tariff implementation index. Water supply and sewerage services.

\section{INTRODUÇÃO}

O acesso aos serviços de água e esgotamento sanitário constitui um elemento essencial à saúde e à vida humana. Apesar dos avanços no setor, o Brasil ainda experiencia um cenário de exclusão sóciosanitária, sobretudo nas regiões em que vivem as populações vulneráveis, onde as desigualdades apresentam-se mais profundas. Nesse contexto, mecanismos de proteção social devem ser operacionalizados de modo a assegurar a tais grupos não somente o acesso a esses importantes serviços do ponto de vista físico, mas, igualmente, sob a perspectiva financeira (COSSENZO, 2013; ONUZIK, et al., 2018;). 
A acessibilidade financeira corresponde à uma das dimensões que compõem os direitos humanos à água e ao esgotamento sanitário (DHAES), reconhecidos pela Assembleia Geral das Nações Unidas e pelo Conselho de Direitos Humanos em julho de 2010 (UNGA, 2010). Pelo prisma dos DHAES, pode-se entendê-la como: o acesso aos serviços de água e esgotamento sanitário de maneira que as pessoas sejam capazes de arcar com os seus custos, satisfazendo os usos pessoais e domésticos da água e garantindo o acesso adequado ao esgotamento sanitário sem comprometer o gozo de demais direitos humanos básicos como, por exemplo, alimentação, moradia, saúde, educação, lazer, entre outros (UNGA, 2010; NEVES-SILVA e HELLER, 2016).

No Brasil, premissas para viabilizar a acessibilidade financeira aos usuários em localidades de baixa renda, podem ser observadas na Lei ${ }^{\circ} 11.445$ de 5 de janeiro de 2007, a qual prevê em seu art. $29, \S$ $2^{\circ}$, a adoção de subsídios inclusivos, tarifários ou não, que garantam o acesso equitativo ao abastecimento de água e ao esgotamento sanitário para todos aqueles com baixa capacidade de pagamento integral das tarifas praticadas pelos prestadores. A forma adotada de proteção à acessibilidade financeira dos usuários considerados como de baixa renda, neste caso, pode ser traduzida por meio das tarifas sociais (BRASIL, 2007; COSSENZO, 2013; BRITTO, 2020).

Em Minas Gerais, os usuários considerados como de baixa renda (renda per capita até meio salário mínimo vigente) possuem, desde o ano de 2011, o direito à TS de água e esgoto nos municípios regulados pela Agência Reguladora de Serviços de Abastecimento de Água e Esgotamento Sanitário do Estado de Minas Gerais (ARSAE - MG). O benefício é concedido às famílias residentes em unidades domiciliares (casa ou apartamento) regulares, sendo a inscrição no Cadastro Único para Programas Sociais do Governo Federal (CadÚnico) pré-requisito indispensável (COPASA, 2012).

Para os usuários atendidos pela Companhia de Saneamento de Minas Gerais (COPASA), o desconto concedido é de $50 \%$ sobre o valor total da fatura. Aqueles que se enquadram nos critérios, mas não usufruem do benefício, devem dirigir-se ao Centro de Referência da Assistência Social (CRAS) mais próximo, solicitar a inscrição no CadÚnico e, posteriormente, comparecer a uma agência de atendimento da Copasa para o cadastramento portando os documentos pessoais, o número de inscrição social (NIS) e a última conta de água e esgoto (COPASA, 2018).

Considerando a relevância das ações voltadas à proteção dos direitos humanos, entre eles os DHAES, com especial atenção às necessidades das populações mais vulneráveis, o presente trabalho objetiva avaliar o Programa de Tarifa Social (PTS) de água e esgoto instituído em municípios atendidos pela COPASA em termos de eficácia e eficiência.

\section{MATERIAIS E MÉTODOS}

Estudo quantitativo, o qual buscou construir a discussão acerca da eficácia e eficiência do PTS na COPASA. Para tanto, obteve-se através da ARSAE-MG uma base de dados contendo o número de economias sociais de água e esgoto referentes aos meses de junho dos anos de 2014 a 2020, para todos os municípios de Minas Gerais cuja prestação dos serviços de abastecimento de água e esgotamento sanitário seja realizada pela COPASA. Não foram incluídos no universo da pesquisa os municípios mineiros atendidos pela subsidiária da COPASA, a Companhia de Serviços de Saneamento Integrado do Norte e Nordeste de Minas Gerais (COPANOR).

Também foi obtido através da ARSAE-MG uma base de dados contendo o número de famílias inscritas no CadÚnico para o mesmo período, que constituía o número de potenciais beneficiários do PTS. Cada base somou 4.088 dados (7 anos para 584 municípios), que foram organizados em 12 grupos conforme a divisão das mesorregiões estabelecidas pelo Instituto Brasileiro de Geografia e Estatística (IBGE) para Minas Gerais: Noroeste de Minas, Norte de Minas, Jequitinhonha, Vale do Mucuri, Triângulo Mineiro e Alto Paranaíba, Central Mineira, Metropolitana de Belo Horizonte, Vale do Rio Doce, Oeste de Minas, Sul e Sudoeste de Minas, Campos das Vertentes e Zona da Mata (IBGE, 
2016). A divisão dos municípios por regiões foi realizada devido a possibilidade de análise mais aprofundada, além de permitir observar quais regiões necessitam de maiores esforços para a plena implementação do PTS. Após a organização dos municípios por região, foi realizado o cálculo do Índice de Implementação da Tarifa Social (IITS), o qual pode ser obtido conforme a equação abaixo:

$$
\text { IITS }=\frac{\text { ESprestador }}{\text { FTSCadÚnico }}
$$

Onde: ESprestador representa o número de economias sociais no banco de faturamento do(s) prestador(es), neste caso, a COPASA; e FTSCadÚnico o número de famílias inscritas no Cadúnico.

O IITS foi construído e adotado pela ARSAE-MG no "Relatório Implementação da Tarifa Social Copasa e Copasa-Copanor - maio de 2020”, e optou-se por reproduzir essa metodologia de cálculo para os demais anos de forma regionalizada, a fim de aprofundar nas análises acerca do PTS. Admitese o cálculo utilizando o FTSCadÚnico como apropriada aproximação do potencial de implementação da tarifa social nos municípios, visto que as famílias com renda per capita de até meio salário mínimo inscritas no CadÚnico podem ser consideradas como uma boa inferência das unidades que deveriam estar cadastradas como economias sociais pelo prestador. Assim, o IITS, dado em porcentagem (\%), consiste na parcela de economias cadastradas como sociais no banco de faturamento da COPASA em relação ao potencial de implementação do benefício que o município apresenta (ARSAE, 2020).

Após o cálculo do IITS para cada município, foram substituídos os casos em que o índice foi superior a $100 \%$, e igualado a $100 \%$, pois não há como o IITS apresentar valores superiores ao máximo. De acordo com esclarecimentos prestados pela ARSAE_MG, as hipóteses possíveis para a explicação desse fato podem ser: cadastro das economias sociais do prestador desatualizado e/ou informações inconsistentes ou incompletas no ato do preenchimento do CadÚnico (ARSAE, 2020).

Após o cálculo de todos os IITS, foram elaborados os gráficos de série histórica do IITS agrupado por região entre os anos de 2014 e 2020. Os gráficos de série histórica foram gerados por meio das médias obtidas por região em cada ano.

\section{RESULTADOS E DISCUSSÃO}

O gráfico apresentado na Figura 1 demonstra a série histórica das médias do Índice de Implementação da Tarifa Social (IITS) de acordo com as mesorregiões de Minas Gerais estabelecidas pelo IBGE, entre os anos 2014 a 2020. Observa-se que as médias do IITS para todas as regiões são superiores a $50 \%$, sendo que a curva da Zona da Mata esteve inferior a todas as outras, apontando que essa é a região onde possivelmente são necessários maiores esforços da COPASA para atualização do cadastro dos usuários beneficiados pelo PTS. Enquanto isso, o IITS da Central Mineira foi o maior de todas as regiões ao longo do tempo. Portanto, infere-se que o PTS foi mais eficaz na região Central Mineira, e menos eficaz na Zona da Mata.

Em regiões como Jequitinhonha e Norte de Minas, o IITS se mantive acima da média em relação a outras regiões, porém, como referido, deve-se destacar que a presente análise não incluiu os municípios atendidos pela subsidiária da COPASA, a COPANOR.

O gráfico apresentado na Figura 2 foi gerado com os mesmos dados da Figura 1, porém, ao invés de linha contínua, exibem-se os marcadores com as médias do IITS para cada região e as linhas de tendência com o comportamento dos dados ao longo do tempo. Dessa forma, observa-se uma tendência de aumento no IITS entre 2015 e 2018 que passou de crescente para decrescente durante o último intervalo de dados (2019-2020). Ao analisar apenas os percentuais, poder-se-ia concluir que o PTS foi mais eficiente entre 2015 e 2018 e menos eficiente entre 2018 e 2019. 

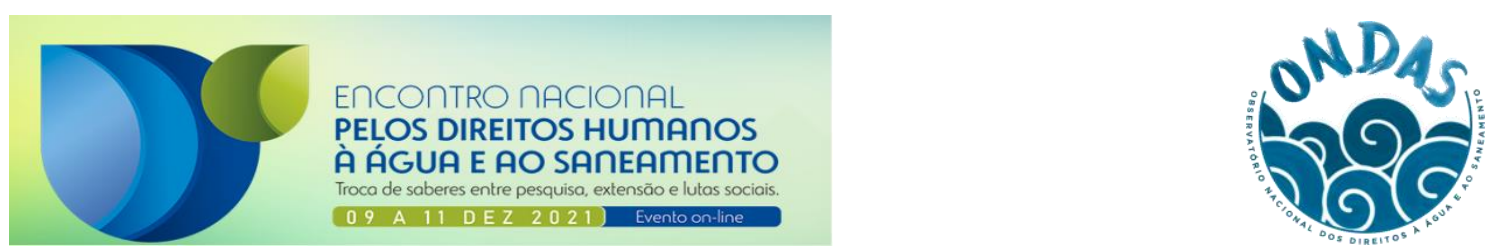

A aparente progressão no IITS entre 2015 e 2018 pode ser, na verdade, consequência dos dados de famílias cadastradas no CadÚnico que não estavam atualizadas, enquanto a queda do IITS observada entre os anos de 2019 e 2020 possivelmente se deve à atualização desses dados, pois anterior à Lei $n^{\circ}$ 23.671/2020, a COPASA era responsável por obter os dados das famílias inscritas no CadÚnico e o fazia através de outras fontes, como: a Caixa Econômica ou por meio dos próprios municípios, o que tornava a tarefa mais trabalhosa.

A desatualização dos dados dos usuários beneficiados pelo PTS se mostrou um dos principais obstáculos em termos de eficácia do Programa, visto que, caso a implementação da tarifa social ocorresse de forma automática, mais usuários cadastrados no CadÚnico seriam contemplados pelo PTS e, consequentemente, o IITS poderia apresentar valores superiores aos apresentados.

Figura 1 - Índice de Implementação da Tarifa Social (IITS) em Água e Esgoto por região de Minas Gerais ao longo do tempo

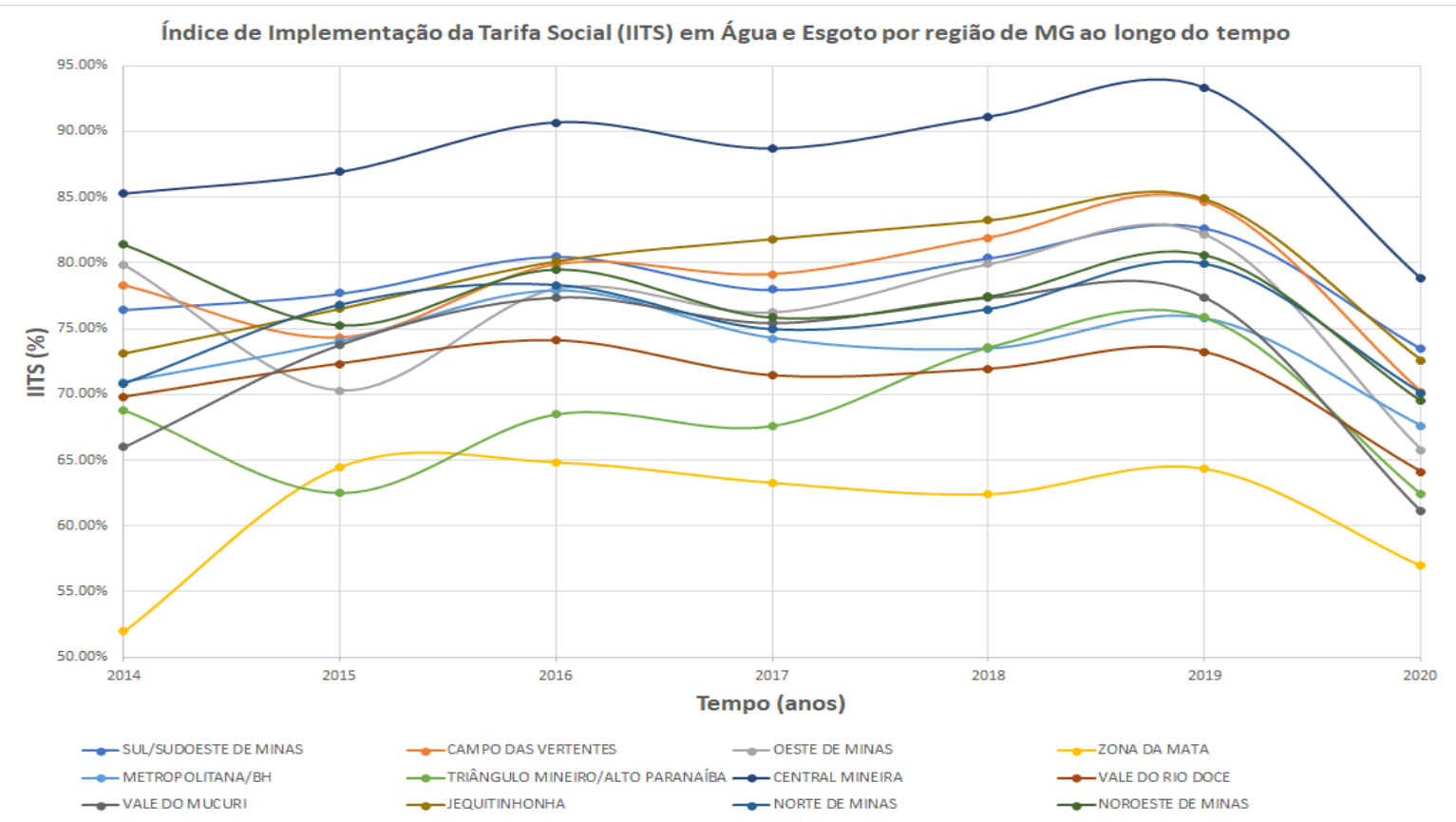

Figura 2 - Linhas de tendência do Índice de Implementação da Tarifa Social (IITS) em Água e Esgoto por região de Minas Gerais ao longo do tempo

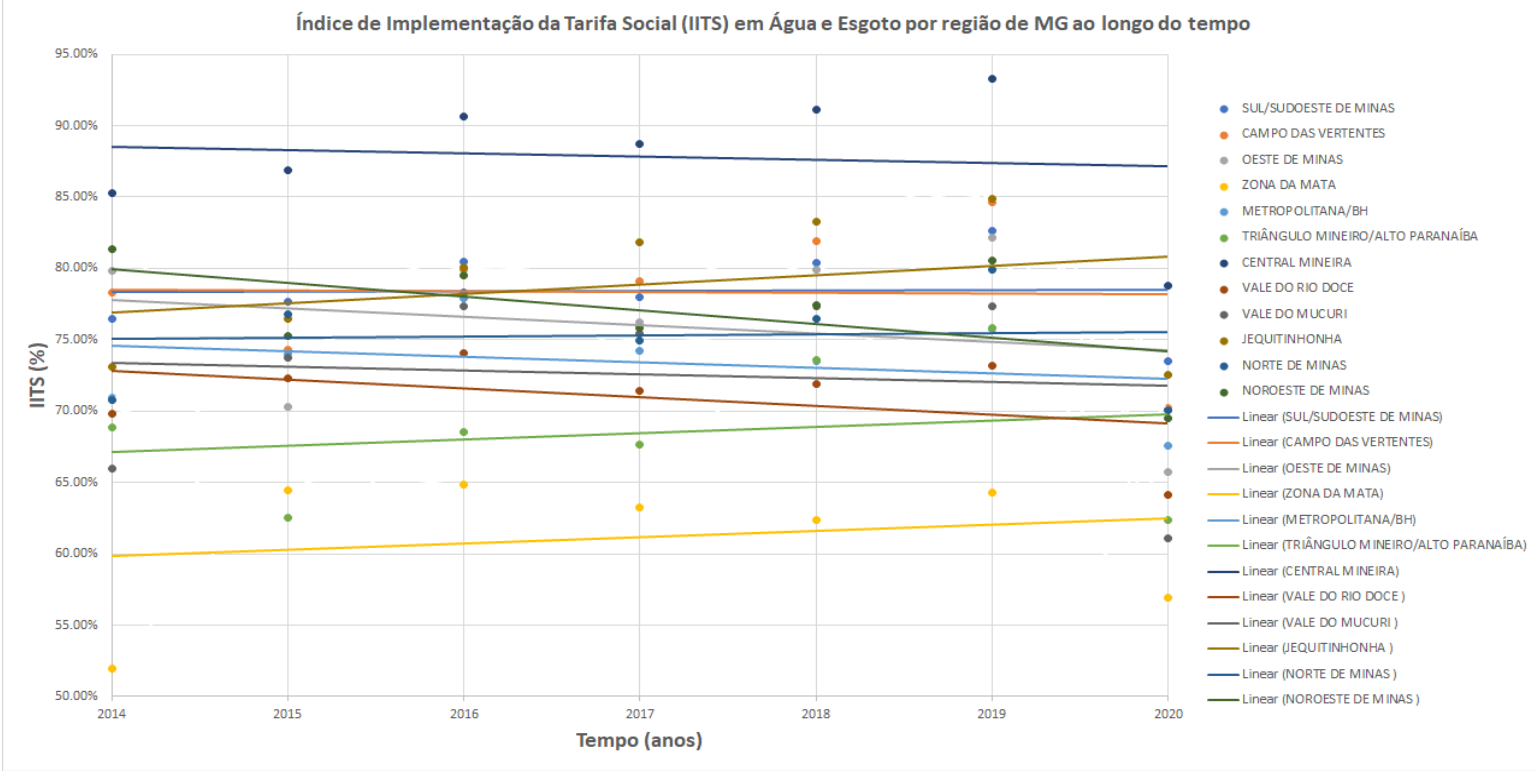


Deve-se destacar que a desatualização desse banco de dados da prestadora de água e esgoto faz com que, não apenas potenciais beneficiários não sejam contemplados pelo PTS, mas também, que famílias que já mudaram de faixa de renda e não estão mais inscritas no CadÚnico continuem usufruindo do benefício, o que explica a existência de municípios com IITS superiores a 100\% (conforme mencionado na metodologia). Nesse sentido, o PTS perde em eficiência, visto que em alguns casos os recursos financeiros para a promoção do PTS foram alocados para subsidiar a tarifa social a usuários que não mais apresentam os requisitos para inclusão no CadÚnico.

\section{CONCLUSÕES}

O IITS varia entre as regiões de Minas Gerais e entre os municípios, existindo municípios com índices abaixo de $20 \%$ cujo valor se dilui quando calculada a média do IITS da região em questão.

A obtenção de dados dos beneficiários do CadÚnico se mostrou um ponto crucial para a implementação automática do benefício da Tarifa Social. A integração entre as políticas de assistência social do estado com as políticas de saneamento desde o início da implementação do PTS poderia ter possibilitado o acesso à COPASA a um banco de dados mais confiável desde 2012 e, portanto, a aplicação mais estratégica do Programa.

A atualização cadastral ocorrida entre os anos de 2019 e 2020 pode favorecer a maior captação de beneficiários elegíveis à tarifa social e contribuir para o crescimento dos IITS e alcance dos objetivos.

Em termos de eficiência, entre 2015 e 2018 observou-se uma progressão no IITS e uma queda entre 2019 e 2020. Já em relação à eficácia, pode-se assumir que as estratégias adotadas pela COPASA caminham na direção da implementação progressiva da tarifa social nos municípios de Minas Gerais.

\section{REFERÊNCIAS}

1. $\quad$ BRASIL. Lei $n^{\circ} 11.445$, de 05 de janeiro de 2007. Estabelece as diretrizes nacionais para saneamento básico. Disponível em: <http://www.planalto.gov.br/ccivil_03/_ato2007-2010/2007/lei/111445.htm>. Acesso em 15 de agosto de 2020.

2. BRITTO, A L. As tarifas sociais de abastecimento de água e esgotamento sanitário no Brasil: seus impactos nas metas de universalização na garantia dos direitos humanos à água e ao esgotamento sanitário. Disponível em: <https://ondasbrasil.org/wpcontent/uploads/2020/05/As-tarifas-sociais-de-abastecimento-de-\%C3\%A1 gua-eesgotamento-sanit\%C3\%A1rio-no-Brasil.pdf>. Acesso em: 21 ago 2020.

3. COSSENZO, C. L. Tarifa social dos serviços de abastecimento de água e esgotamento sanitário no Distrito Federal. Dissertação (Mestrado). Universidade de Brasília.

4. INSTITUTO BRASILEIRO DE GEOGRAFIA E ESTATÍSTICA (INGE). Lista das Mesorregiões de Minas Gerais. Disponível em: https://www.mg.gov.br/sites/default/files/paginas/arquivos/2016/ligminas_10_2_04_listame somicro.pdf. Acesso em out 2020.

5. NEVES-SILVA, P; HELLER, L. O direito humano à água e ao esgotamento sanitário como instrumento para promoção da saúde de populações vulneráveis. Ciênc. saúde coletiva, Rio de Janeiro, v. 21, n. 6, p. 1861-1870, 2016.

6. NOTA TÉCNICA 05/2012: Detalhamento do cálculo do reajuste tarifário da Companhia de Saneamento de Minas Gerais, COPASA-MG, e das tarifas a serem aplicadas a partir de maio de 2012. Disponível em: 


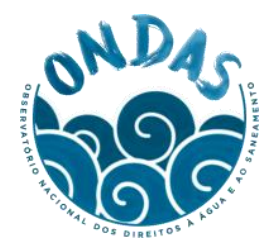

http://www.arsae.mg.gov.br/images/documentos/nota_tecnica_05_2012_reajuste_copasa_20 12.pdf. Acesso em 03 out 2020.

7. NOTA TÉCNICA GRT No 09/2018: Detalhamento do Cálculo do Reajuste Tarifário de 2018 da Companhia de Saneamento de Minas Gerais (COPASA). Disponível em: http://www.arsae.mg.gov.br/images/documentos/audiencia_publica/NT_GRT_09_Reajuste Copasa_2018.pdf. Acesso em 03 out 2020.

8. ONUZIK, N C; TAVARES, R ; LIMA, C A M ; FAUSTO, M A . Programa Bolsa Família - Uma análise sob a óptica das condições socioambientais em que vivem as famílias beneficiárias em um município da Serra do Espinhaço, MG, Brasil. Revista de Estudos Ambientais (online), v. 20, p. 58-69, 2018.

9. UNITED NATIONS GENERAL ASSEMBLY. Human Right to Water and Sanitation. UM Document A/RES/64/292. Geneva: UNGA, 2010.

\section{LIMITAÇÕES DO ESTUDO}

Apesar da obtenção de um extenso banco, a fragilidade dos dados obtidos foi uma das limitações deste trabalho, a qual refletiu na incerteza acerca das análises do IITS. Questiona-se se de fato houve progressão entre os anos de 2015 e 2018, ou se esse comportamento pode ser oriundo da desatualização dos dados. Para fins de futuras publicações sobre o tema, os autores pretendem entrar em contato com a COPASA de modo a realizar entrevistas, dado que não foi possível obter retorno da prestadora em tempo hábil para a realização deste trabalho.

\section{AGRADECIMENTOS}

Os autores agradecem à ARSAE-MG o apoio recebido por meio do compartilhamento da base de dados utilizada para as análises. 